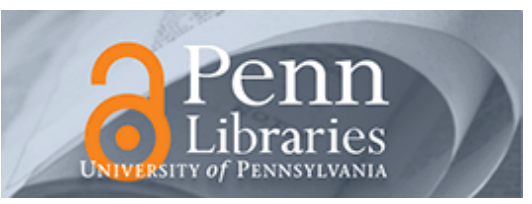

University of Pennsylvania

ScholarlyCommons

September 2005

\title{
Dielectrophoretic Assembly of Carbon Nanofiber Nanoelectromechanical Devices
}

\author{
Stephane Evoy \\ University of Alberta \\ Michael A. Riegelman \\ University of Pennsylvania \\ Nevin Naguib \\ Drexel University \\ Haihui Ye \\ Drexel University \\ Papot Jaroenapibal \\ Drexel University \\ Follow this and additional works at: https://repository.upenn.edu/mse_papers
}

See next page for additional authors

Recommended Citation

Evoy, S., Riegelman, M. A., Naguib, N., Ye, H., Jaroenapibal, P., Luzzi, D. E., \& Gogotsi, Y. (2005).

Dielectrophoretic Assembly of Carbon Nanofiber Nanoelectromechanical Devices. Retrieved from

https://repository.upenn.edu/mse_papers/76

Copyright 2005 IEEE. Reprinted from IEEE Transactions on Nanotechnology, Volume 4, Issue 5, September 2005, pages 570-575.

This material is posted here with permission of the IEEE. Such permission of the IEEE does not in any way imply IEEE endorsement of any of the University of Pennsylvania's products or services. Internal or personal use of this material is permitted. However, permission to reprint/republish this material for advertising or promotional purposes or for creating new collective works for resale or redistribution must be obtained from the IEEE by writing to pubs-permissions@ieee.org. By choosing to view this document, you agree to all provisions of the copyright laws protecting it.

This paper is posted at ScholarlyCommons. https://repository.upenn.edu/mse_papers/76

For more information, please contact repository@pobox.upenn.edu. 


\title{
Dielectrophoretic Assembly of Carbon Nanofiber Nanoelectromechanical Devices
}

\begin{abstract}
We report a technique for the assembly of bottom-up nanomechanical devices. This technique employs the dielectrophoretic manipulation of nanostructures within a multiple layer lithography process. Mechanical resonators were specifically produced by assembling and clamping tubular carbon fibers onto prefabricated pads. Our preliminary results showed that an assembled cantilevered fiber with length $\mathrm{L}=5 \mu \mathrm{m}$ and width of $\mathrm{W}=180 \mathrm{~nm}$ possessed a resonant frequency of $f=1.17 \mathrm{MHz}$. A shorter $\mathrm{L}=3-\mu \mathrm{m}-$ long singly clamped resonator of similar width showed a resonance of $f=3.12 \mathrm{MHz}$. This frequency range is in agreement with the low gigapascal bending moduli previously reported for carbon structures showing extensive volume defects. This technology would allow the integration of bottom-up nanostructures with other more established fabrication processes, thus allowing the deployment of engineered nanodevices in integrated systems.

\section{Keywords}

Detectors, materials processing, microelectromechanical systems, microresonators, nanotechnology

Comments

Copyright 2005 IEEE. Reprinted from IEEE Transactions on Nanotechnology, Volume 4, Issue 5, September 2005, pages 570-575.

This material is posted here with permission of the IEEE. Such permission of the IEEE does not in any way imply IEEE endorsement of any of the University of Pennsylvania's products or services. Internal or personal use of this material is permitted. However, permission to reprint/republish this material for advertising or promotional purposes or for creating new collective works for resale or redistribution must be obtained from the IEEE by writing to pubs-permissions@ieee.org. By choosing to view this document, you agree to all provisions of the copyright laws protecting it.
\end{abstract}

\section{Author(s)}

Stephane Evoy, Michael A. Riegelman, Nevin Naguib, Haihui Ye, Papot Jaroenapibal, David E. Luzzi, and Yury Gogotsi 


\title{
Dielectrophoretic Assembly of Carbon Nanofiber Nanoelectromechanical Devices
}

\author{
Stephane Evoy, Michael A. Riegelman, Nevin Naguib, Haihui Ye, Papot Jaroenapibal, David E. Luzzi, and \\ Yury Gogotsi
}

\begin{abstract}
We report a technique for the assembly of bottom-up nanomechanical devices. This technique employs the dielectrophoretic manipulation of nanostructures within a multiple layer lithography process. Mechanical resonators were specifically produced by assembling and clamping tubular carbon fibers onto prefabricated pads. Our preliminary results showed that an assembled cantilevered fiber with length $L=5 \mu \mathrm{m}$ and width of $W=180 \mathrm{~nm}$ possessed a resonant frequency of $f=1.17 \mathrm{MHz}$. A shorter $L=3$ - $\mu \mathrm{m}$-long singly clamped resonator of similar width showed a resonance of $f=3.12 \mathrm{MHz}$. This frequency range is in agreement with the low gigapascal bending moduli previously reported for carbon structures showing extensive volume defects. This technology would allow the integration of bottom-up nanostructures with other more established fabrication processes, thus allowing the deployment of engineered nanodevices in integrated systems.
\end{abstract}

Index Terms-Detectors, materials processing, microelectromechanical systems, microresonators, nanotechnology.

\section{INTRODUCTION}

$\mathbf{T}$ HE LAST several decades have seen a phenomenal growth in the availability of computational power and communications capacity. A second microelectronics revolution is underway, defined by the integration of transistor-based electronics with micromechanical and nanomechanical actuators, micropumps, and valves, as well as physical, biological, and chemical sensors. These integrated systems are poised to receive, process, and distribute large quantities of data concerning the physical world, and offer great interest for the development of distributed sensor networks in a wide range of commercial and military applications. Nanometer scale research and development provide control over materials at the level of individual atoms and molecules. At these lengths lie the basic phenomena that determine health versus disease,

Manuscript received July 6, 2004; revised January 21, 2005. This work was supported by the National Science Foundation (NSF) under Award ECS-0225439. The work of M. A. Riegelman, N. Naguib, H. Ye, and Y. Gogotsi was supported by the NSF under the Nanoscale Interdisciplinary Research Team Program CTS-0210579.

S. Evoy was with the Department of Electrical and Systems Engineering, The University of Pennsylvania, Philadelphia, PA 19104 USA. He is now with the Department of Electrical and Computer Engineering, University of Alberta, Edmonton, AB T6G 2V4, Canada.

M. A. Riegelman is with the Department of Mechanical Engineering and Applied Mechanics, The University of Pennsylvania, Philadelphia, PA 19104 USA.

N. Naguib, H. Ye, P. Jaroenapibal, D. E. Luzzi, and Y. Gogotsi are with the Department of Materials Science and Engineering, Drexel University, Philadelphia, PA 19104 USA.

Digital Object Identifier 10.1109/TNANO.2005.851404 govern chemical reactions, control electronic behavior, and determine materials strength. Such nanometer scale engineering offers new approaches for the implementation of materials and devices that would offer key attributes of low-cost, low power consumption, and high sensitivity in integrated systems.

The manipulation of individual nanostructures is necessary for the characterization of their electrical and mechanical behavior, the analysis of their response to outside agents and stimuli, and, more importantly, their eventual integration into single-chip systems. Following the pioneering work of Pohl [1], we and others have used dielectrophoretic (DEP) forces to manipulate and assemble nanostructures from liquid suspensions. Dielectrophoresis is defined as the motion of uncharged polarizable particles in a nonuniform electric field. These forces were used to trap biomaterials such as single-virus particles, capsids, and latex spheres [2]. More recently, dielectrophoeretic manipulation enabled transport measurements across individual DNA strands trapped within nanometer-scale electrode gaps [3]. The DEP assembly of single-walled carbon nanotubes (SWNTs) has also been reported, demonstrating its viability for the assembly of such materials [4]-[6].

While dielectrophoresis presents demonstrated potential for the manipulation of nanostructures, it also offers the important advantage of being compatible with standard microelectronics foundry technologies, eventually allowing the on-chip assembly and integration of nanodevices with transduction, readout, signal processing, and communications circuitry. We have proposed a novel integrated systems technology based on the DEP assembly of nanostructures (Fig. 1). We have obtained a preliminary proof of this concept by successfully positioning one-dimensional metallic nanowires onto prefabricated sites of silicon circuitry, and have reported the preliminary integration of such structures with CMOS chips [7]. Cantilever-based devices specifically represent promising platforms for high-performance sensing devices. Sensors based on a mechanical resonating element enable the frequency modulation of the output, thus greatly improving the stability/noise-immunity of the reading [8]. This report, therefore, describes the fabrication and characterization of mechanical resonators produced through the DEP assembly of tubular carbon nanofibers.

\section{EXPERIMENTAL}

Experiments were performed using commercially available Pyrograph tubular carbon nanofibers purchased from Applied Sciences Inc., Cedarville, $\mathrm{OH}$. The fibers were grown by catalytic chemical vapor deposition (CVD) using methane as a 


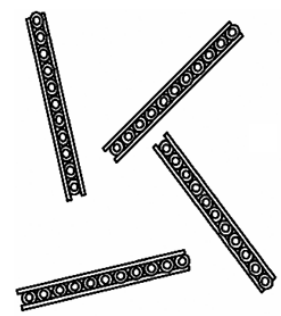

a)

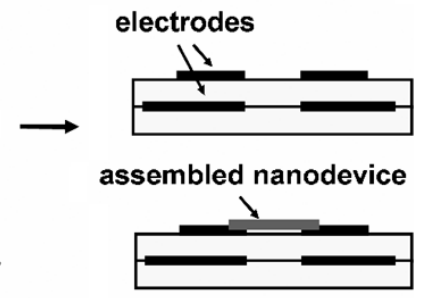

b)

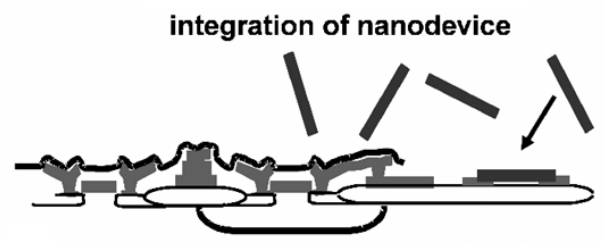

c)

Fig. 1. DEP integration of nanodevices. (a) Functional structures are designed using "bottom-up" synthesis techniques. (b) Dielectrophoresis allows the inclusion of these structures within a multilayer fabrication process and (c) their integration with prefabricated processing circuitry.

precursor. The material was then heat treated at $3000{ }^{\circ} \mathrm{C}$ to graphitize the carbon and remove the catalyst, suspended in isopropanol at a concentration of $0.1 \mathrm{mg} / \mathrm{mL}$, and ultrasoniced for $30 \mathrm{~min}$. The solution was then filtered through a $10-\mu \mathrm{m}$ micropore membrane (Osmonics Inc., Minnetonka, MN) to dispose of the shorter fibers. The fibers were re-suspended in isopropanol at a concentration of $0.1 \mu \mathrm{g} / \mathrm{mL}$, and ultrasoniced for an additional $15 \mathrm{~min}$ immediately prior to assembly. Structural inspection of the material was performed using a JEOL 2010F transmission electron microscope (TEM). The end material consisted of a distribution of tubular nanofibers with diameters ranging from $D=75$ to $200 \mathrm{~nm}$, lengths ranging from $L=5$ to $15 \mu \mathrm{m}$, and wall thicknesses ranging from $t=20$ to $40 \mathrm{~nm}$.

Using an approach reported by Smith et al. [9], individual fibers were dielectrophoretically positioned onto capacitively coupled electrode pairs fabricated on a silicon wafer (Fig. 2). First, a 300-nm electrically insulating $\mathrm{SiO}_{2}$ layer was grown using thermal oxidation at $1000{ }^{\circ} \mathrm{C}$. A first set of electrodes was patterned out of a 170-nm layer of $\mathrm{Au}$, with an underlying 30-nm layer of $\mathrm{Cr}$, using photolithography and liftoff. The electrodes were then buried under $300 \mathrm{~nm}$ of CVD $\mathrm{SiO}_{2}$. Top floating electrodes with gap spacings varying from 1 to $5 \mu \mathrm{m}$ were then fabricated in a similar metal layer [see Fig. 2(a)]. The top electrodes are capacitively driven by feeding a $45-\mathrm{V}_{p p} 100-\mathrm{kHz}$ assembly signal between the sets of buried electrodes for $5 \mathrm{~min}$. This signal was provided by a Topward 8110 function generator, and amplified by a Bogen GA-6A amplifier [see Fig. 2(b)]. Following assembly, a final electron beam lithography step is performed to deposit a 200-nm-thick "clamping" layer onto the extremities of selected structures using the assembly electrodes themselves as alignment marks. The nanofibers are then released by etching down the underlying oxide layers in a 10:1 buffered HF for $45 \mathrm{~s}$ [see Fig. 2(c)].

Preliminary mechanical assaying was performed using an interferometric method originally developed for the characterization of surface machined silicon nanoelectromechanical

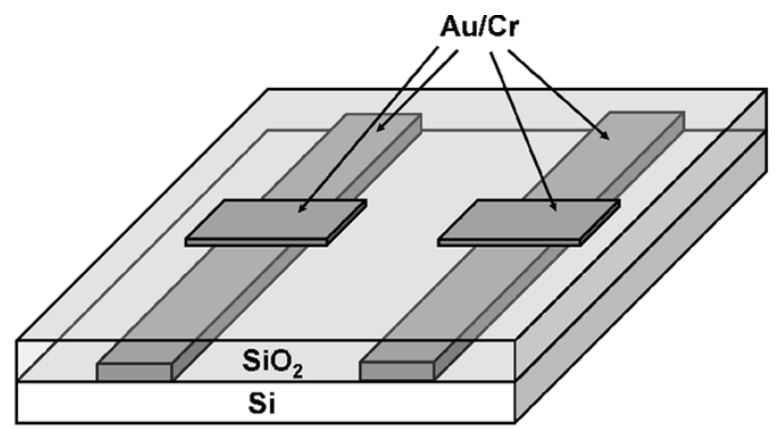

a)

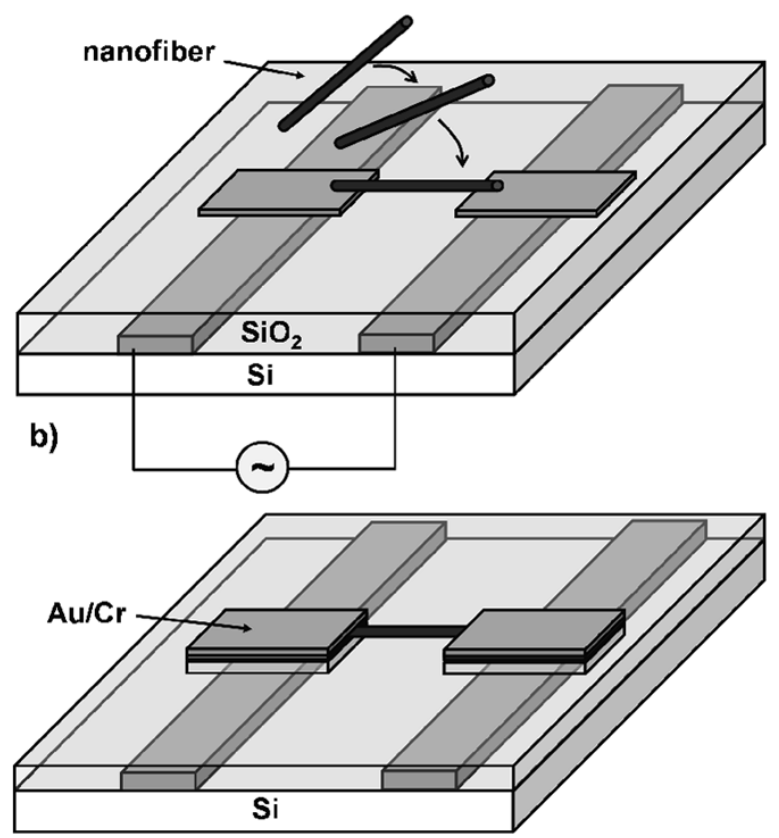

c)

Fig. 2. DEP assembly of carbon nanotube resonators. (a) Assembly is performed on microfabricated pads capacitively coupled to buried electrodes. (b) A $100-\mathrm{kHz}$ signal is applied between the buried electrodes to induce the assembly of the tubes across the top pads. (c) Structures are clamped using post-assembly lithography and released by partial etching of the oxide layer.

systems (NEMS) resonators [10], [11]. The chip is mounted onto a small piezoelectric element (Radio Shack 273-059), which is inserted in a small vacuum chamber pumped down to the $10^{-3}$-torr range [see Fig. 3(a)]. The piezoelectric element is then actuated by the tracking output of a Hewlett-Packard ESA-L1500A spectrum analyzer amplified by an ENI UA-400A power amplifier. An He-Ne laser $(\lambda=633 \mathrm{~nm})$ was focused onto the nanofiber device using a 0.35 -NA microscope objective. When actuated at resonance, relative motion of the structure with respect to the underlying substrate modulates the reflected signal through interferometric effects [see Fig. 3(b)]. The modulated signal is reflected back through the microscope objective. A beam splitter is then employed to divert the reflected signal toward a New Focus 1601 ac coupled photodetector, whose output is fed to the input of the spectrum analyzer. While the diameter of the resonating structures is substantially smaller than the laser wavelength, their surfaces induce sufficient amount of optical scattering and interference with an underlying substrate to generate a reflected optical signal that is discernible by the photodetector. The same technique 


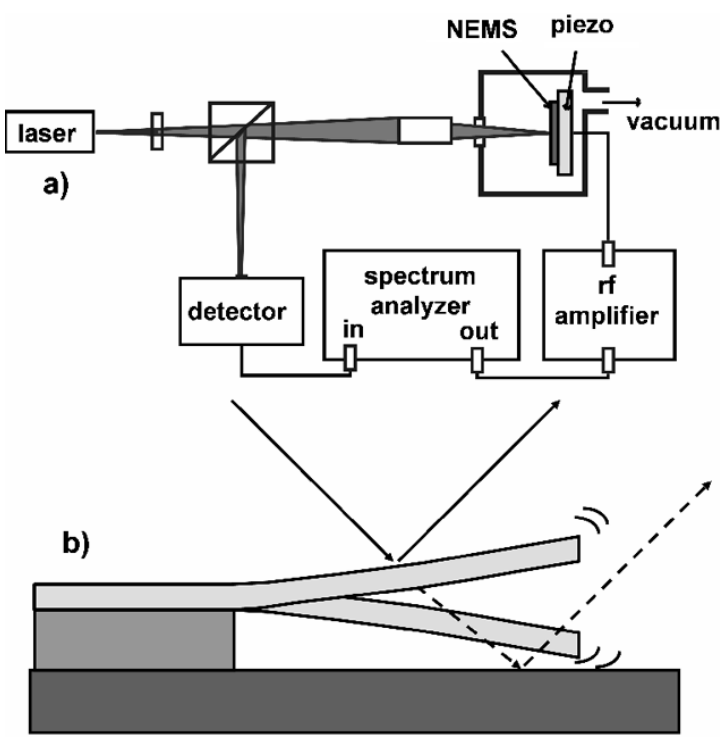

Fig. 3. Schematic diagram of interferometric setup employed for nanomechanical characterization of assembled resonators. (b) Resonant cantilever induces modulation of reflected signal through interference with underlying substrate.

has indeed previously been demonstrated for the assaying of surface machined resonators with lateral dimensions as small as $50 \mathrm{~nm}[11]$.

\section{RESULTS AND DISCUSSION}

Fig. 4 shows TEM micrographs of typical fibers that have been directly deposited from solution onto a sample grid. The tubular fibers show diameters ranging from $D=75 \mathrm{~nm}$ to $200 \mathrm{~nm}$, and wall thicknesses from $t=20 \mathrm{~nm}$ to $40 \mathrm{~nm}$. Some fibers also show bamboo-shaped features [see Fig. 4(a)], which have previously been observed in large-diameter tubes grown by CVD [12]-[14], and can be regarded as stacking of truncated conical graphene sheets [13]. A higher resolution TEM image [see Fig. 4(b)] reveals the finer detail of this conical scroll structure, which is similar to the one described in [15]. Finally, the same image shows that tube surface also possesses a "herringbone" wall structure and chemically stable arched edges [16] that were induced by the $3000{ }^{\circ} \mathrm{C}$ heat treatment employed to graphitize the carbon and remove the catalyst.

Fig. 5 shows a typical nanofiber dielectrophoretically assembled across a $2-\mu \mathrm{m}$ gap. This particular micrograph was taken prior to the clamping and release of the resonator. At its extremity, the nanofiber shows an outer diameter $D=200 \mathrm{~nm}$. However, the tube cross section shows partial compression that rather results in ribbon-shaped geometry. Additional inspection by scanning electron microscopy (SEM) confirmed the dominance of such ribbon-shaped geometry both prior and after assembly. Such deformation is common for large-diameter nanotubes having thin walls [17].

While fibers are successfully assembled across a majority of the gaps, the reliability of this approach is first limited by our ability to trap a single structure free from obstruction arising from the assembly of additional fibers in a same gap. Nonetheless, roughly $10 \%$ of the assembly gaps indeed contained a single well-positioned fiber with significant amount
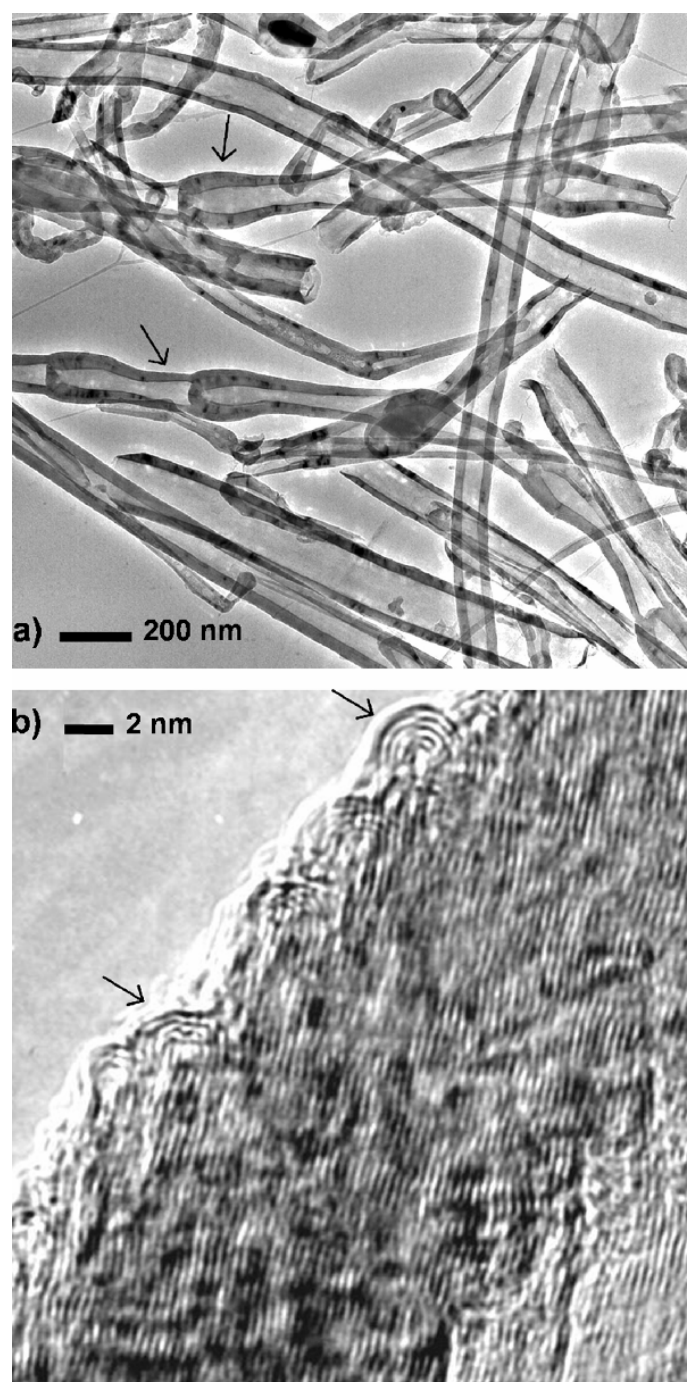

Fig. 4. Transmission electron micrographs of heat-treated carbon tubes. (a) Tubes nominally possess an outer diameter ranging from $D=100 \mathrm{~nm}$ to $200 \mathrm{~nm}$, and a wall thickness ranging from $t=25$ to $50 \mathrm{~nm}$. Some tubes show bamboo-shaped structural features (arrows). (b) The tube outer surfaces also show chemically stable arched "herringbone" edges (arrows).

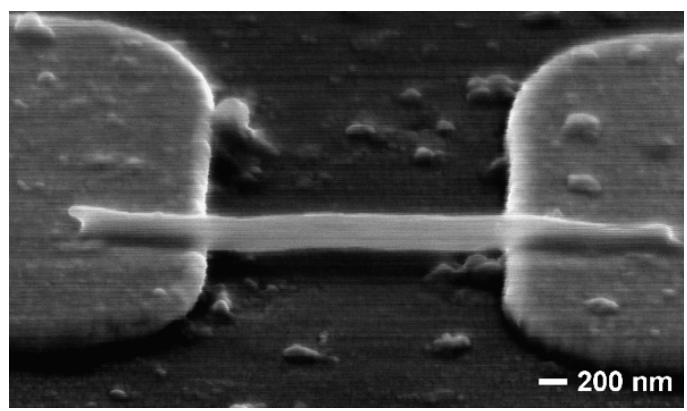

Fig. 5. SEM of assembled carbon nanofiber.

of clamping length on either or both electrodes. In addition, throughput of the technique is further restricted by the inclination of the assembled structures to stick to the underlying surface upon release. Such issue could be greatly alleviated by using a critical point drying process in order to avoid the structures from being pulled down to the substrate during drying. 


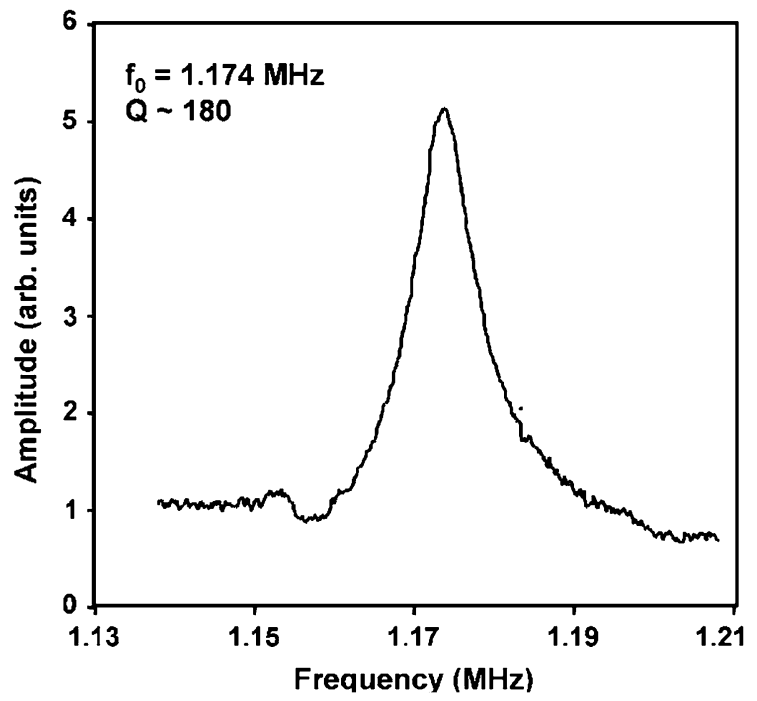

Fig. 6. Frequency response or assembled resonator of length $L=5 \mu \mathrm{m}$ and width $W=180 \mathrm{~nm}$.

Nonetheless, a number of the straighter and thicker fibers were successfully released, thus allowing a preliminary assessment of mechanical properties.

Fig. 6 shows the resonant spectrum of a released singleclamped nanofiber. The 7- $\mu \mathrm{m}$-long and 180-nm-wide fiber was assembled and clamped on one extremity with a free length $L=5 \mu \mathrm{m}$. The structure showed a resonant frequency of $f=1.17 \mathrm{MHz}$. A similar structure of similar width showing a free length of $3 \mu \mathrm{m}$ possessed a fundamental resonance of $f=3.12 \mathrm{MHz}$. Resonance qualities of $Q \sim 150-180$ were determined from the width of the Lorentzian frequency response. These qualities are not necessarily intrinsic to the fibers, but could be affected by combination of clamping-point dissipation and viscous damping due to the moderate vacuum employed.

The following relationship between resonant frequency and dimensions is derived from the Euler-Bernoulli analysis of a prismatic cantilevered beam [18]:

$$
f_{i}=\frac{B_{i}^{2}}{2 \pi L^{2}} \sqrt{\frac{E^{*} I}{\rho A}}
$$

with

$$
I=\frac{\pi\left(D_{o}^{4}-D_{i}^{4}\right)}{64}
$$

and

$$
A=\frac{\pi\left(D_{o}^{2}-D_{i}^{2}\right)}{4}
$$

where $f_{i}$ is the resonant frequency, $L$ is the length, $I$ is the cross-sectional moment of inertia of a cylindrical tube, $A$ is its area, $D_{o}$ is its external diameter, $D_{i}$ is its internal diameter, $E^{*}$ is the effective bending modulus, $\rho$ is the density of graphite $\left(\rho=2.22 \mathrm{~g} / \mathrm{cm}^{3}\right)$ [19], and $B_{i}$ is a constant for the $i$ th harmonic oscillation $B_{1}=1.875, B_{2}=4.694$.

The bending modulus of carbon tubes has been experimentally assessed by several techniques such as tensile loading tests [20], [21], observation of thermally induced vibrations [22], and in situ actuation in the TEM [23]. While the reported Young's moduli of SWNTs are typically around a terapascal, such values tend to be significantly lower in carbonaceous structures of increasing diameters due to increasing dominance of point defects and structural imperfections. More specifically, Poncharal et al. observed effective bending moduli in the $E^{*} \sim 200 \mathrm{GPa}$ range for $\sim 20-40$-nm-diameter multiwall carbon nanotubes (MWNTs) grown by an arc discharge method [23]. However, the same group then observed effective bending moduli as low as $E^{*} \sim 2-3 \mathrm{GPa}$ in structurally imperfect MWNTs produced by precursor pyrolisis, and concluded that point and volume defects played a dominant role in the reduction of the overall bending modulus [24]. Deformation of the tube cross section is also expected to lower the position of resonance in these fibers. Indeed, resonant frequency is a strong function of the transverse dimension of the beam through the cross-sectional moment of inertia. Departure from a cylindrical geometry to a more elliptical shape would significantly reduce the moment of inertia of the fiber along the bending axis. For instance, a cylindrical nanotube with outer diameter of $D_{0}=180 \mathrm{~nm}$ and wall thickness of $t=30 \mathrm{~nm}$ subject to a partial collapse that would reduce its transverse dimension $20 \%$ while keeping same cross-sectional area through similar lateral extension would see its bending moment of inertia reduced by as much as $40 \%$. Using an effective bending modulus of $E^{*}=2-3 \mathrm{GPa}$, the expected resonances of such fibers would range between 0.8-1.5 MHz for a 5- $\mu \mathrm{m}$-long structure, and between 2.3-3.7 $\mathrm{MHz}$ for a 3- $\mu \mathrm{m}$-long fiber, which is in rough agreement with the observed quantities.

In summary, the range of resonant frequencies of the dielectrophoretically assembled nanofibers resonators is in agreement with the low gigapascal bending moduli previously reported for carbonaceous tubes showing extensive volume defects [24]. Such characteristics could, therefore, be intrinsic to the conical scrolls or bamboo-shaped fibers being assembled. In addition, the partial structural collapse observed in these tubes would also reduce their resonant frequencies by decreasing their bending moment of inertia. A more systematic assessment of these properties would, however, require structures showing better uniformity and of simpler cross-sectional geometry.

\section{CONCLUSION}

We have reported a novel technique for the assembly of "bottom-up" nanoelectromechanical devices. DEP manipulation allowed the positioning of nanostructures at specific sites of a prefabricated pattern, enabling the integration of the structures within a multiple layer lithography process. Specifically, released devices were produced by assembling tubular carbon fibers onto prefabricated pads, followed by their clamping through a post-assembly electron beam lithography step. Preliminary mechanical resonances in the low megahertz range were observed in singly clamped $3-5-\mu \mathrm{m}$-long cantilevered fibers. A more conclusive assessment of mechanical properties would, however, require structures showing better uniformity and of simpler cross-sectional geometry. This technology would allow the integration of bottom-up nanostructures with other more established fabrication processes, thus allowing the deployment of nanostructured devices in integrated systems. 


\section{REFERENCES}

[1] H. A. Pohl, Dielectrophoresis: The Behavior of Neutral Matter in NonUniform Electric Fields. New York: Cambridge Univ. Press, 1978.

[2] M. Hughes and H. Morgan, "AC electrokinetics: A review of forces in microelectrode structures," J. Phys. D, Appl. Phys., vol. 31, pp. 2205-2210, 1998.

[3] D. Porath, A. Bezryadin, S. de Vries, and C. Dekker, "Direct measurement of electrical transport through DNA molecules," Nature, vol. 403 , pp. 635-638, 2000.

[4] L. A. Nagahara, I. Amlani, J. Lewenstein, and R. K. Tsui, "Directed placement of suspended carbon nanotubes for nanometer-scale assembly," Appl. Phys. Lett., vol. 80, pp. 3826-3828, 2002.

[5] R. Krupke, F. Hennrich, H. B. Weber, M. M. Kappes, and H. Lohneysen, "Simultaneous deposition of metallic bundles of single-walled carbon nanotubes using AC-dielectrophoresis," Nano Lett., vol. 3, pp. 1019-1023, 2003.

[6] S. W. Lee, D. S. Lee, H. Y. Yu, E. E. B. Campbell, and Y. W. Park, "Production of individual suspended single-walled carbon nanotubes using the AC electrophoresis technique," Appl. Phys. A, vol. A78, pp. 283-286, 2004.

[7] S. Evoy et al., "Dielectrophoretic assembly and integration of nanowire devices with functional CMOS operating circuitry," Micro. Eng., vol. 75, pp. 31-42, 2004

[8] R. R. A. Syms, "Electrothermal frequency tuning of folded and coupled vibrating micromechanical resonators," J. Microelectromech. Syst., vol. 7, no. 2, pp. 164-171, Jun. 1998.

[9] P. A. Smith, C. D. Nordquist, T. N. Jackson, T. S. Mayer, B. R. Martin, J. Mbindyo, and T. E. Mallouk, "Electric-field assisted assembly and alignment of metallic nanowires," Appl. Phys. Lett., vol. 77, pp. 1399-1401, 2000.

[10] D. W. Carr, L. Sekaric, and H. G. Craighead, "Measurement of nanomechanical resonant structures in single-crystal silicon," J. Vac. Sci. Technol. B, Microelectron. Process. Phenom., vol. 16, pp. 3821-3824, 1998.

[11] D. W. Carr, S. Evoy, L. Sekaric, H. G. Craighead, and J. M. Parpia, "Measurement of mechanical resonance and losses in nanometer scale silicon wires," Appl. Phys. Lett., vol. 75, pp. 920-922, 1999.

[12] N. A. Kiselev, J. Sloan, D. N. Zakharov, E. F. Kukovitskii, J. L. Hutchison, J. Hammer, and A. S. Kotosonov, "Carbon nanotubes from polyethylene precursors: Structure and structural changes caused by thermal and chemical treatment revealed by HREM," Carbon, vol. 36, pp. 1149-1157, 1998.

[13] Z. Li, J. Chen, X. Zhang, Y. Li, and K. K. Fung, "Catalytic synthesized carbon nanostructures from methane using nanocrystalline Ni," Carbon, vol. 40, pp. 409-415, 2002.

[14] C. J. Lee, J. Park, and J. A. Yu, "Catalyst effect on carbon nanotubes synthesized by thermal chemical vapor deposition," Chem. Phys. Lett., vol. 360 , pp. $250-255,2002$

[15] H. Ye, N. Naguib, Y. Gogotsi, A. G. Yazicioglu, and C. M. Megaridis, "Wall structure and surface chemistry of hydrothermal carbon nanofibers," Nanotechnology, vol. 15, pp. 232-236, 2004.

[16] L. Chen, H. Ye, and Y. Gogotsi, "Synthesis of boron nitride coating on carbon nanotubes," J. Amer. Ceram. Soc., vol. 87, pp. 147-151, 2004.

[17] M. P. Rossi, H. Ye, Y. Gogotsi, S. Babu, P. Ndungu, and J.-C. Bradley, "Environmental scanning electron microscopy study of water in carbon nanopipes," Nano Lett., vol. 4, pp. 989-993, 2004.

[18] L. Meirovich, Elements of Vibration Analysis. New York: McGrawHill, 1986.

[19] H. O. Pierson, Handbook of Carbon, Graphite, Diamond and Fullerenes. Park Ridge, NJ: Noyes, 1994.

[20] E. W. Wong, P. E. Sheehan, and C. M. Lieber, "Nanobeam mechanics: Elasticity, strength and toughness of nanorods and nanotubes," Science, vol. 277, pp. 1971-1975, 1997.

[21] J. P. Salvetat, G. A. D. Briggs, J.-M. Bonard, R. R. Bacsa, A. J. Kulik, T. Stockli, N. A. Burnham, and L. Forro, "Elastic and shear moduli of single-walled carbon nanotube ropes," Phys. Rev. Lett., vol. 82, pp. 944-947, 1999.

[22] A. Krishnan, E. Dujardin, T. W. Ebbessen, P. N. Yianilos, and M. M. J. Treacy, "Young's modulus of single-walled nanotubes," Phys. Rev. B, Condens. Matter, vol. 58, pp. 14013-14 019, 1998.

[23] P. Poncharal, Z. L. Wang, D. Ugarte, and W. A. de Heer, "Electrostatic deflections and electromechanical resonances of carbon nanotubes," Science, vol. 283, pp. 1513-1516, 1999.

[24] R. Gao, Z. L. Wang, Z. Bai, W. A. de Heer, L. Dai, and M. Gao, "Nanomechanics of individual carbon nanotubes from pyrolytically grown arrays," Phys. Rev. Lett., vol. 85, pp. 622-625, 2000.

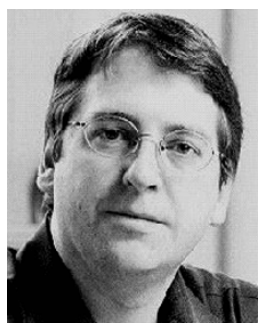

Stephane Evoy received the B.Eng. and M.Sc.A. degrees in engineering physics from the Ecole Polytechnique of Montreal, Montreal, QC, Canada, in 1992 and 1994, respectively, and the Ph.D. degree in applied physics from Cornell University, Ithaca, NY, in 1998.

From 1998 to 1999, he was Post-Doctoral Associate with the Laboratory of Atomic and Solid State Physics, Cornell University. From 1999 to 2001, he was an Assistant Professor with the Department of Electrical and Computer Engineering, Virginia Polytechnic Institute and State University. From 2002 to 2004, he was an Assistant Professor with the Department of Electrical and Systems Engineering, The University of Pennsylvania. Since August 2004, he has been an Assistant Professor of electrical and computer engineering with the Department of Electrical and Computer Engineering, University of Alberta, Edmonton, AB, Canada, and a Researcher with the Canadian National Research Council (NRC) National Institute for Nanotechnology (NINT). His current research interests include the development and integration of nanomechanical and nanofluidic devices and the biofunctionalization of nanomechanical resonators for applications in proteomic assaying.

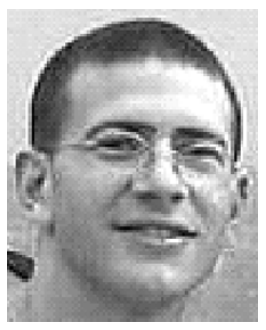

Michael A. Riegelman received the B.S. degree in mechanical engineering from George Washington University, Washington, DC, in 2002, the M.S. degree in mechanical engineering from The University of Pennsylvania, Philadelphia, in 2004 respectively, and is currently working toward the Ph.D. degree in mechanical engineering at The University of Pennsylvania.

From 2002 to 2004, he conducted research in several areas including RF microelectromechanical systems (MEMS), microfluidics/nanofluidics, and electrokinetic flows and manipulations. He was also with the Army Research Laboratory as a Clean-Room Technician and with the Naval Research Laboratory as a Summer Intern. He currently conducts research on molecular motors with the National Institutes of Health (NIH) under the NIH's Graduate Partnership Program.

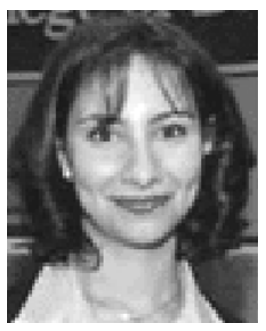

Nevin Naguib received the B.S. degree in chemistry (with the highest distinction and college honors) from the University of Illinois at Urbana-Champaign, in 2000, and the M.S. degree in materials science and engineering from Drexel University, Philadelphia, PA, in 2004.

In July 2004, she accepted a post-doctoral position with the Materials Science Division, Argonne National Laboratory, Argonne, IL. Her interests include modification of carbon nanotubes and nanostructures as a first step to their usage in potential applications such as nanofluidic devices and nanocomposite reinforcements.

Ms. Naguib has been the recipient of several awards including Best Poster Awards at Drexel University Research Day and by the Philadelphia Chapter of ASM International. She was also the recipient of a National Science Foundation (NSF)- IGERT Fellowship (2002 and 2003), a Dean's Fellowship, and the Koerner Fellowship (2001).

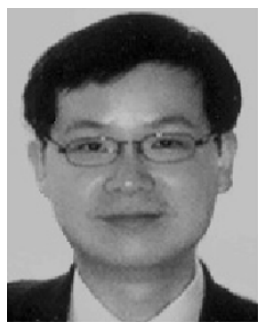

Haihui Ye received the Ph.D. degree in materials science from the Max Planck Institute for Metals Research, University of Stuttgart, Stuttgart, Germany, in 2002.

$\mathrm{He}$ is currently a Research Associate with the A. J. Drexel Nanotechnology Institute, Drexel University, Philadelphia, PA. His research interests include carbon nanomaterials such as carbon nanotubes, fullerenes and nanodiamonds, carbon-based nanocapsules, supercapacitors, biosensors, nanofluidic devices and drug delivery systems, surface functionalization and atomistic processes at surfaces, and composite nanowires and thin films. His expertise includes transmission electron microscopy (TEM), electron energy loss spectroscopy, energy-filtered TEM imaging, and various other nanocharacterization techniques. 


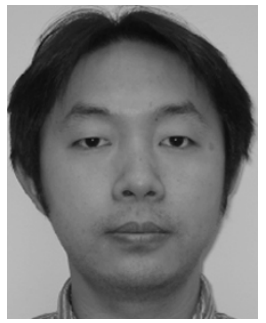

Papot Jaroenapibal received the B.S. degree in materials science and engineering from Columbia University, New York, NY, in 2002, the M.S. degree in materials science and engineering from The University of Pennsylvania, Philadelphia, in 2005, and is currently working toward the Ph.D. degree in materials science and engineering at The University of Pennsylvania. His doctoral dissertation specifically concerns in situ TEM studies of mechanical properties of carbon nanotubes, hybrid nanotubes, and nanowire systems.

His research interests include fabrication and characterization of carbon nanotube-based electromechanical devices.

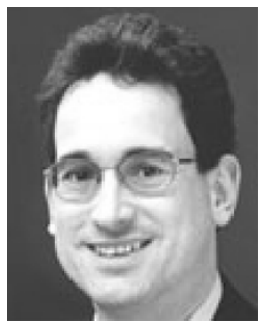

David E. Luzzi received the B.E. degree in engineering physics from the Stevens Institute of Technology, Hoboken, NJ, in 1980, and the Ph.D. degree in materials science from Northwestern University, Evanston, IL, in 1986.

Since 1987, he has been a Professor of materials science with The University of Pennsylvania, Philadelphia. From 1996 to 1997, he was also a Visiting Professor with Kyoto University. He has authored over 100 papers in peer-reviewed journals. His research projects in nanoscale systems are focused on materials processing and applications of functionalized nanotubes and nanoparticles.

Dr. Luzzi is a member of the Air Force Scientific Advisory Board. He was a science and technology advisor for the Chief of Naval Operations Strategic Studies Group. He is a Fellow of the Defense Science Study Group. He was a past-president of the Philadelphia Society for Microscopy. He was the recipient of the 2002 George Heilmeier Award for Research Innovation.

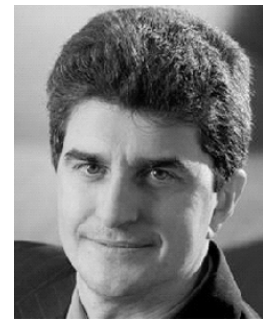

Yury Gogotsi received the M.S. and Ph.D. degrees from Kiev Polytechnic, Kiev, Ukraine, in 1984 and 1986, respectively, and the D.Sc. degree from the Ukrainian Academy of Science, Kiev, Ukraine, in 1995.

$\mathrm{He}$ is currently a Professor of materials science and engineering with the Department of Materials Science and Engineering, Drexel University, Philadelphia. He also holds appointments with the Departments of Chemistry and Mechanical Engineering, Drexel University. He is the Director of the A. J. Drexel Nanotechnology Institute and Associate Dean of the College of Engineering, Drevel University. His research group studies carbon nanotubes, nanostructured carbons, nanofluidics, and pressure-induced phase transformations in ceramics and semiconductors. He has coauthored two books, edited six books, and authored over 200 research papers. He holds 20 patents.

Dr. Gogotsi is a Fellow of the American Ceramic Society, a member of the World Academy of Ceramics, and a member of the International Institute for the Science of Sintering. He was the recipient of several awards for his research including the I. N. Frantsevich Prize presented by the Ukrainian Academy of Science, the S. Somiya Award presented by the International Union of Materials Research Societies, the Kuczynski Prize presented by the International Institute for the Science of Sintering, and the Roland B. Snow Award presented by the American Ceramic Society. 